\title{
The Arteriolar Glycocalyx Plays a Role in the Regulation of Blood Flow in the Iliac of the Anaesthetised Pig
}

\author{
T. RUANE-O'HORA ${ }^{1}$, F. MARKOS ${ }^{1}$ \\ ${ }^{1}$ Department of Physiology, University College Cork, Cork, Ireland
}

Received March 6, 2017

Accepted August 24, 2017

On-line November 10, 2017

\section{Summary}

The role of the glycocalyx of arterial resistance vessels in regulating blood flow in vivo is not fully understood. Therefore, the effect of glycocalyx damage using two separate compounds, hyaluronidase and N-Formylmethionyl-leucyl-phenylalanine (fMLP), was evaluated in the iliac artery vascular bed of the anaesthetised pig. Blood flow and pressure were measured in the iliac, an adjustable snare was applied to the iliac above the pressure and flow measurement site to induce step decreases (3 occlusions at 3-4 min intervals were performed for each infusion) in blood flow, and hence iliac pressure, and vascular conductance (flow/pressure) was calculated. Saline, hyaluronidase (14 and $28 \mu \mathrm{g} / \mathrm{ml} / \mathrm{min})$, and fMLP (1 $\mu \mathrm{M} / \mathrm{min}$ ) were infused separately, downstream of the adjustable snare and their effect on arterial conductance assessed. Hyaluronidase at the higher infusion rate and FMLP both caused a reduction in arterial conductance, and hence an increase in blood flow resistance. In conclusion, the results show that glycocalyx damage causes an increase in resistance to blood flow in the iliac artery vascular bed.

\section{Key words}

Glycocalyx • N-Formylmethionyl-leucyl-phenylalanine (fMLP), • Hyaluronidase $\bullet$ Blood flow

\section{Corresponding author}

F. Markos, Department of Physiology, University College Cork, Cork, Ireland. E-mail: f.markos@ucc.ie

\section{Introduction}

The endothelial glycocalyx lines the inner surface of all blood vessels in the body and is a complex structure composed of proteoglycans, glycoproteins and associated glycosaminoglycans (Tarbell et al. 2014). Functionally, it has been reported to play an important role in vascular physiology, including acting as a mechanotransducer for blood flow (Reitsma et al. 2007, Tarbell et al. 2014). There is also evidence that the glycocalyx is altered/damaged in a number of pathophysiological conditions, including hyperglycaemia/diabetes, sepsis, atherosclerosis, hypertension and cancer (Tarbell and Cancel 2016). Most recent vascular studies have tended to focus on the glycocalyx lining larger blood vessels, both arterial and venous, with little mention of what role the arteriolar-resistance vessel glycocalyx might play. Pries et al. (1997) conducted a study on rat mesenteric arteries in vivo and found that heparinase infusion caused a significant fall in blood flow resistance, whereas neuraminidase treatment caused an increase in resistance. This study indicated that the glycocalyx contributes to blood flow regulation; however, the results also show that the effect on resistance depends on which component of the glycocalyx was damaged. Whether the arteriolar glycocalyx as a whole increases or decreases blood flow is not clear. Therefore, in the present study the effect of glycocalyx damage using two separate compounds, hyaluronidase and N-Formylmethionyl-leucyl-phenyl-alanine (fMLP), was studied in a large animal in vivo preparation designed to assess its role in altering resistance artery diameter (Markos et al. 2014, Ruane-O'Hora et al. 2013).

\section{Methods}

This investigation was carried out under licenses 
issued by the Department of Health, Ireland and the Health Products Regulatory Authority, as directed by the Cruelty to Animals Act Ireland, updated legislation, and European Union statutory instructions.

\section{Surgery and instrumentation}

Twelve Landrace pigs (18.4-24.2 kg) were sedated with ketamine $(14 \mathrm{mg} / \mathrm{kg})$ and xylazine $(2.7 \mathrm{mg} / \mathrm{kg})$ i.m. A cannula was inserted into an ear vein and the animal was anesthetized with a bolus (induction, $30 \mathrm{mg} / \mathrm{kg}$ i.v.), followed by a continuous infusion (Harvard Apparatus, Cambridge, UK) of sodium pentobarbital (maintenance, $6 \mathrm{mg} / \mathrm{kg} / \mathrm{h}$ i.v.) via a catheter inserted into the jugular vein. End-tidal carbon dioxide $\left(\mathrm{ETCO}_{2}\right)$, pulse oximetry and core temperature were monitored using SurgiVet AdvisorVital Signs Monitor (Smiths Medical, Dublin, $\mathrm{OH}$ ). Arterial pH, $\mathrm{PCO}_{2}$ and $\mathrm{PO}_{2}$ were assessed using an i-STAT blood gas analyzer (Abbot Point of Care Inc, Princeton, NJ) and maintained within their normal ranges. Following tracheotomy animals were ventilated with $40 \% \mathrm{O}_{2}$ in room air using a Harvard Apparatus ventilation pump at a rate adjusted to keep end-tidal and arterial $\mathrm{PCO}_{2}$ within a normal range (35-40 $\mathrm{mm} \mathrm{Hg})$. A cannula attached to a pressure transducer (Grass; Grass Technologies, West Warwick, RI) was inserted into the left carotid artery for measurement of arterial blood pressure and to obtain arterial samples. The left or right iliac artery was dissected from the aortic bifurcation to the deep femoral branch, and a cannula attached to a 3-way tap was inserted into the deep femoral artery for infusions of saline, hyaluronidase, or N-Formylmethionyl-leucylphenylalanine (fMLP) downstream of the flow and pressure measurement sites in the iliac. In two pigs a branch of the deep femoral was cannulated and a hyaluronidase infusion experiment was conducted. Pressure in the iliac was measured using a catheter tipped manometer (Millar, Houston, Texas, USA) placed in a side branch; an ultra-sonic transit time flow transducer (Transonic Systems Inc., Ithaca, New York, USA) was placed around the iliac to measure blood flow. An adjustable snare was placed below the iliac-aorta bifurcation, above the pressure and flow measurement sites, to produce controlled restrictions to blood flow. Data was recorded using Power lab pre-amplifiers and software (AD Instruments Ltd, Oxford, UK) and a Dell computer. Following experimental procedures, animals were killed using a lethal intravenous injection of pentobarbitone and $\mathrm{KCl}$.

\section{Experimental protocol}

Intra-arterial infusions distal to the iliac pressure and flow measurement sites (in 10 pigs via the deep femoral artery and in 2 pigs a branch of the deep femoral) were made of, saline alone at the same flow rate as the intervention, then hyaluronidase at 14 and subsequently $28 \mu \mathrm{g} / \mathrm{ml} / \mathrm{min}$. Or, saline followed by fMLP at $1 \mu \mathrm{M} / \mathrm{ml} / \mathrm{min}$. Only one infusion experiment, either hyaluronidase or fMLP, per iliac was conducted. While the infusion was maintained, the proximal snare was used to restrict blood flow once every four minutes (a total of 3 occlusions per infusion protocol were carried out) as previously described (Markos et al. 2014, Ruane-O'Hora et al. 2013).

\section{Drugs used}

Hyaluronidase and AMLP were obtained from Sigma (Wicklow, Ireland). All drugs were dissolved in physiological saline before injection.

\section{Statistical analysis}

Data is expressed as mean \pm S.E. Vascular conductance was obtained from the slope of the relationship between flow and pressure $(\Delta \mathrm{F} / \Delta \mathrm{P})$. The slopes from the interventions were compared to the control slope, using homogeneity of regressions analysis (SPSS), only if the slope value was significant (ANOVA; Excel). $\mathrm{P}<0.05$ was considered significant.

\section{Results}

\section{Hyaluronidase}

Seven pigs were used in this series of experiments, and prior to the onset of the experiment heart rate averaged $134 \pm 11$ beats $/$ min, mean arterial blood pressure was $100 \pm 8 \mathrm{~mm} \mathrm{Hg}$, and mean iliac artery blood flow averaged $105 \pm 14 \mathrm{ml} / \mathrm{min}$.

Figure 1 summarises the data from this experiment. The control conductance value (slope), following the occlusions during the saline infusion protocol, was $0.43 \pm 0.17 \mathrm{ml} / \mathrm{min} / \mathrm{mm} \mathrm{Hg}$ which was statistically significant ( $\mathrm{P}=0.019$, ANOVA). Infusing hyaluronidase at $14 \mu \mathrm{g} / \mathrm{ml} / \mathrm{min}$ resulted in a conductance of $0.36 \pm 0.21 \mathrm{ml} / \mathrm{min} / \mathrm{mm} \mathrm{Hg}$ which was not by itself significant $(\mathrm{P}=0.1, \mathrm{ANOVA})$. The subsequent higher infusion rate of $28 \mu \mathrm{g} / \mathrm{ml} / \mathrm{min}$ further reduced the conductance to $0.19 \pm 0.25$ which again was not a significant value ( $\mathrm{P}=0.44$, ANOVA). In conclusion, the data shows that hyaluronidase infusion at the higher rate 
causes a large reduction in vascular conductance in the vascular beds supplied by the iliac.

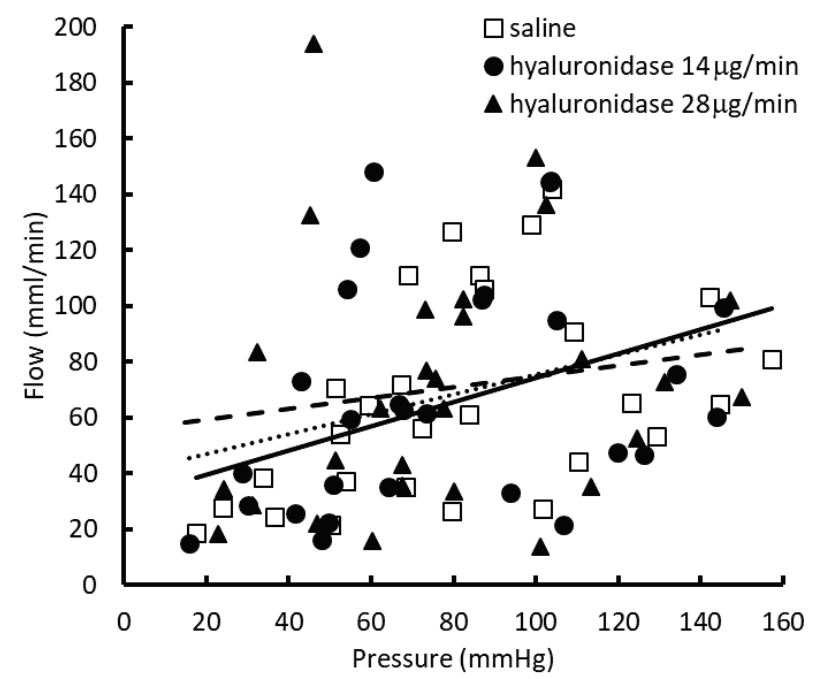

Fig. 1. The effect of hyaluronidase infusion on iliac vascular conductance. Hyaluronidase infusion at the higher dose (dashed line) caused a greater decrease in conductance than the lower dose (dotted regression line) when compared to saline infusion (solid line).

\section{$f M L P$}

Six pigs were used in this series of experiments, at the beginning of the experiment heart rate averaged $129 \pm 12$ beats/min, mean arterial blood pressure was $86 \pm 5 \mathrm{~mm} \mathrm{Hg}$, and mean iliac artery blood flow averaged $120 \pm 13 \mathrm{ml} / \mathrm{min}$.

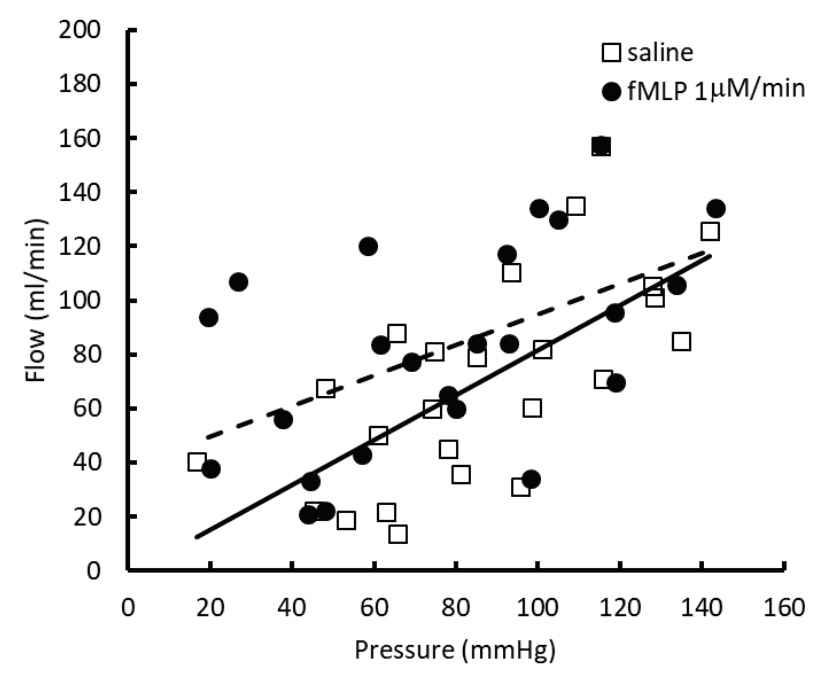

Fig. 2. The effect of N-Formylmethionyl-leucyl-phenylalanine (fMLP) infusion on iliac vascular conductance. fMLP infusion (dashed line) caused a significant decrease in conductance when compared to saline infusion (solid line).
The control conductance was $0.83 \pm 0.19 \mathrm{ml} / \mathrm{min} / \mathrm{mm} \mathrm{Hg}$ which was statistically significant $(\mathrm{P}<0.001$, ANOVA). $\mathrm{fMLP} 1 \mu \mathrm{M} / \mathrm{ml} / \mathrm{min}$ gave a reduced slope of $0.57 \pm 0.2 \mathrm{ml} / \mathrm{min} / \mathrm{mm} \mathrm{Hg}$ which was significant ( $\mathrm{P}=0.009$, ANOVA) (Fig. 2). As both slope values were significant, they were compared using homogeneity of regressions analysis which indicated that the reduction in conductance due to AMLP infusion was significantly different to the control saline infusion $(\mathrm{P}<0.001)$.

\section{Discussion}

The results of this study indicate that arteriolar glycocalyx damage causes an increase in arterial vascular resistance in the iliac artery vascular bed in vivo. Two separate compounds were used to degrade the glycocalyx, enzymatic cleavage of hyaluronic acid using hyaluronidase; and fMLP, which leads to an immune response known to cause damage to all components of the glycocalyx (Gao and Lipowsky 2010). The initial dose of hyaluronidase used is known to significantly reduce shear-induced nitric oxide (NO) production from isolated perfused canine femoral arteries after 20 min continuous exposure (Mochizuki et al. 2003). Further, unpublished immunohistochemical data from our laboratory showed that this concentration $(14 \mu \mathrm{g} / \mathrm{ml})$ and duration of exposure caused a greater than $50 \%$ reduction in hyaluronic acid expression in the pig iliac glycocalyx. In the present study, the first infusion rate only caused a slight reduction in conductance, it required the subsequent doubling of the infusion dose to greatly reduce vascular conductance; suggesting a cumulative effect of hyaluronidase. The most likely reason an additional dose, and hence extra time, was required to fully uncover the effect of hyaluronidase was that and in the present study blood flow was not restricted. Most previous experiments either used closed in vitro preparations, using excised arteries or cultured cells, or isolated arteries in vivo where blood containing enzyme was left to act (unpublished pig artery data from our laboratory). It should be noted that Gao and Lipowsky (2010) used a continuous blood flow model for their hyaluronidase infusion experiment. However, their study was focused on the venous glycocalyx in the rat mesentery and not a large animal systemic circulation.

Similarly, fMLP infusion at a dose used previously in the rat (Gao and Lipowsky 2010, Lipowsky et al. 2011) in vivo also caused a significant fall in 
vascular conductance in the vascular beds supplied by the pig iliac. Again, the rat studies were conducted on the venous side of the circulation; more significantly, the most notable difference between our study and the rat studies was the route of fMLP action. In rats, fMLP was applied topically onto exteriorized mesenteric tissue and not via a direct luminal infusion. These superfusion studies have shown the effect of fMLP to be time dependent, with shedding of the glycocalyx starting as early as 5 min after superfusion began, and continuing for the $30 \mathrm{~min}$ period of measurement (Lipowsky et al. 2011). In our study, each iliac artery infusion protocol lasted for approximately $15 \mathrm{~min}$, although one major limitation of the present pig study is that we are unable to visualise or quantify the extent of glycocalyx damage in the resistance vessels downstream of the iliac. In fact, it is difficult to identify and hence instrument resistance arteries in vivo (Christensen and Mulvany 2001).

In conclusion, the results from this study suggest that the glycocalyx plays a role in the regulation of arterial blood flow, and consequently blood pressure, in vivo. Further work is required to assess whether this finding is of significance in a variety of disease states which directly affect the vascular glycocalyx.

\section{Conflict of Interest}

There is no conflict of interest.

\section{Acknowledgements}

SurgiVet Monitor purchased with a grant from the Strategic Research Fund UCC 2012

\section{References}

CHRISTENSEN KL, MULVANY MJ: Location of resistance arteries. J Vasc Res 38: 1-12, 2001.

GAO L, LIPOWSKY HH: Composition of the endothelial glycocalyx and its relation to its thickness and diffusion of small solutes. Microvasc Res 80: 394-401, 2010.

LIPOWSKY HH, GAO L, LESCANIC A: Shedding of the endothelial glycocalyx in arterioles, capillaries, and venules and its effect on capillary hemodynamics during inflammation. Am J Physiol Heart Circ Physiol 301: H2235-H2345, 2011.

MARKOS F, SHORTT CM, EDGE D, RUANE-O'HORA T, NOBLE MIM: Immediate direct peripheral vasoconstriction in response to hyperinsulinaemia and metformin in the anaesthetised pig. Physiol Res 63: 559-566, 2014.

MOCHIZUKI S, VINK H, HIRAMATSU O, KAJITA T, SHIGETO F, SPAAN JA, KAJIYA F: Role of hyaluronic acid glycosaminoglycans in shear-induced endothelium-derived nitric oxide release. Am J Physiol Heart Circ Physiol 285: H722-H726, 2003.

PRIES AR, SECOMB TW, JACOBS H, SPERANDIO M, OSTERLOH K, GAEHTGENS P: Microvascular blood flow resistance: Role of the endothelial surface layer. Am J Physiol 273: H2272-H2279, 1997.

REITSMA S, SLAAF DW, VINK H, VAN ZANDVOORT MA, OUDE EGBRINK MG: The endothelial glycocalyx: composition, functions and visualization. Pflugers Arch 454: 345-359, 2007.

RUANE-O'HORA T, EDGE D, SHORTT CM, MARKOS F, NOBLE MIM: Responses of iliac conduit artery and hindlimb resistance vessels to luminal hyperfructosaemia in the anaesthetized pig. Acta Physiol (Oxf) 209: 254-261, 2013.

TARBELL JM, SIMON SI, CURRY FR: Mechanosensing at the vascular interface. Annu Rev Biomed Eng 16: 505-532, 2014.

TARBELL JM, CANCEL LM: The Glycocalyx and it significance in human medicine. J Intern Med 280: 97-113, 2016. 